\title{
STEM EBIC Thermometry Calibration with PEET on Al Nanoparticles
}

William Hubbard ${ }^{1}$, Matthew Mecklenburg ${ }^{2}$ and B. C. Regan $^{3}$

${ }^{1}$ NanoElectroning Imaging, Inc. (NEI), Los Angeles, California, United States, ${ }^{2}$ University of Southern California, Los Angeles, California, United States, ${ }^{3}$ UCLA Department of Physics \& Astronomy, Los Angeles, California, United States

TEM is the tool of choice for high-resolution imaging of electronic devices. However, standard TEM imaging contrast, even with spectroscopic attachments, is primarily sensitive to physical and chemical properties. These properties are sometimes of only secondary interest compared to electronic and thermal properties, especially for operating devices. Advances in scanning TEM electron beam induced current (STEM EBIC) imaging have enabled mapping of electronic properties in live devices, including resistance, electric field, potential, and work function, at high spatial resolution [1-3]. We have also observed a thermal response in the STEM EBIC signals from operating devices. Here we compare this temperature response with independent, TEM-based thermometry in an effort to quantify the thermal dependence of these EBIC signals.

Other recently developed TEM-based thermometry techniques extract temperature from plasmon [4], phonon [5,6], or diffraction [7] measurements. Of these, only plasmon energy expansion thermometry (PEET) [4] has mapped nanoscale thermal gradients. PEET has the additional advantage of being able to measure temperature in common materials, such as Al. In this work we use PEET thermometry on Al nanoparticles to calibrate the STEM EBIC temperature response.

EBIC imaging is the association of the current generated in a sample with a scanned electron beam's position. In the "standard" EBIC mode, current is generated by the separation of electron-hole pairs in local electric fields. A more recently demonstrated EBIC mode, SEEBIC, measures current produced in a sample by the emission of secondary electrons [1]. We have observed temperature-dependent signals in both standard EBIC and SEEBIC. The signal's magnitude increases with temperature for both modes.

We have fabricated samples for measuring the temperature response in both EBIC modes via simultaneous PEET and STEM EBIC measurements. In both architectures, a Pt heater is fabricated on a $20 \mathrm{~nm}$-thick silicon nitride membrane framed by a Si substrate [1]. SEEBIC thermometry samples (Fig. 1) place the heater adjacent to an amorphous carbon electrode. In standard EBIC samples (not shown) the heater is adjacent to a capacitor structure, where a voltage applied to the bottom electrode produces a large standard EBIC signal, which is measured from the top electrode. For both sample architectures Al nanoparticles are drop-cast on the entire sample to act as PEET nanothermometers.

For the Fig. 1 device, PEET and SEEBIC are acquired at the edge of the carbon electrode in a region of the membrane (yellow box in the Fig. 1 ADF image) which contains areas of carbon, Al, Al on carbon, and bare membrane. For each heater bias value, a STEM EBIC image and an electron energy loss spectroscopy (EELS) spectrum image are acquired in this region. EELS spectra at each pixel are fit [4] to obtain PEET temperature values from the Al nanoparticles. The Fig. 1 plot shows SEEBIC from the carbon region, normalized to the average room temperature SEEBIC values, as a function of the PEET-derived temperature. Each heater power is applied in both bias polarities. The measured EBIC signal is independent of the bias polarity, indicating its thermal origin. The SEEBIC signal shows a roughly quadratic response to temperature. 
EBIC thermometry is particularly appealing for measuring temperature in operating electronic devices. Any TEM-compatible device that is electrically connected for in situ operation already satisfies many of the challenging requirements for compatibility with STEM EBIC. Unlike PEET, STEM EBIC does not require the complicated fitting of 3D datasets, and it is relatively agnostic to the specific materials used for thermometry as long as the device architecture provides a measurable EBIC signal. The existence of a temperature signal in the different EBIC modes adds to the versatility of the thermometry technique. For example, the SEEBIC heating platform shown in Fig. 1 exhibits no standard EBIC, while EBIC acquired from a biased gate in a transistor will be dominated by standard EBIC, but STEM EBIC thermometry can be performed in both cases [8].
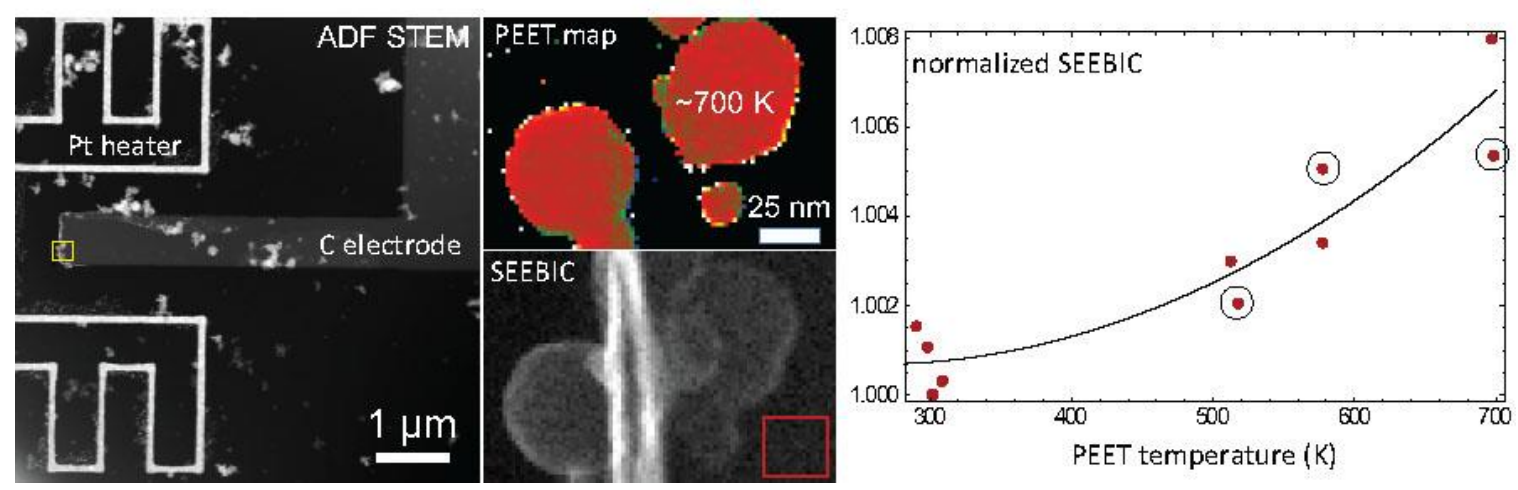

Figure 1. (Left) ADF image of Pt heater and amorphous carbon electrode with Al nanoparticles dispersed across on sample to act as PEET thermometers. (Middle) PEET and SEEBIC maps simultaneously acquired in the yellow box in the ADF image while the heater is energized. This region contains large $\mathrm{Al}$ nanoparticles on (right) and off (left) the carbon electrode. Note that both nanoparticles measure the same $\sim 700 \mathrm{~K}$ temperature in the field of view. The plot on the right shows signal in the bottom-right red box of the SEEBIC image, normalized to the average of the room temperature measurements, as a function of temperature, as measured on the Al nanoparticles. Circled points indicate data taken under negative heater bias polarity. The black line represents a quadratic fit to the data meant to be a guide to the eye (rather than a rigorous characterization of the data).

\section{References}

[1] WA Hubbard et al., Physical Review Applied 10 (2018), p. 044066.

[2] WA Hubbard et al., Applied Physics Letters 115 (2019), p. 133502.

[3] M Mecklenburg et al., Ultramicroscopy 207 (2019), p. 112852.

[4] M Mecklenburg et al., Science 347 (2015), p. 629-632.

[5] M Lagos et al., Nano Letters 18 (2018), p. 4556-4563.

[6] J C Idrobo et al., Physical Review Letters 120 (2018), p. 095901.

[7] G Wehmeyer et al., Applied Physics Letters 113 (2018), p. 253101.

[8] This material is based upon work supported by the Defense Microelectronic Activity under Contract No. HQ072720P0004, and by NSF STC award DMR-1548924 (STROBE), NSF award DMR-1611036, and the UCLA PSEIF. 